Acta Cryst. (2002). A58 (Supplement), C67

POSSIBILITY OF CONVERTING A CHYMOTRYPSIN INHIBITOR TO A TRYPSIN INHIBITOR

A. Seal J. Dattagupta

Inter University Consortium For DAE Facility, Kalkuta, India

It has been found that all Kunitz type inhibitors of serine proteases have same reactive site confirmation and have reasonable sequence identity.Structure of a Kunitz type chymotrypsin inhibitor WCI was solved at C\&MB Division of SINP,Kolkata,India.The walls of the bindofing pockets of Chymotrypsin and Trypsin can also be aligned within root-mean-square deviation of less than $0.6 \AA$ A. So,we explored the idea of converting a chymotrypsin inhibitor to a trypsin inhibitor by mutating the key residue with the appropriate residue of trypsin inhibitor. From modeling studies using INSIGHT II program package,we conclude that ARG at key residue might serve better purpose than LYS

Keywords: KUNITZ TYPE, CHYMOTRYPSIN INHIBITOR, DOCKING
Acta Cryst. (2002). A58 (Supplement), C67

STRUCTURE-BASED HIGH THROUGHPUT VIRTUAL SCREENING FOR DRUG DISCOVERY

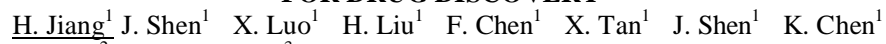
I. Silman $^{2}$ J. L. Sussman ${ }^{3}$

1=Center for Drug Discovery and Design, Shanghai Institute of Materia Medica, Chinese Academy of Sciences, Shanghai 200031, China 2=Department of Neurobiology, Weizmann Institute of Science, Rehovot 76100, ISRAEL 3=Department of Structural Biology, Weizmann Institute of Science, Rehovot 76100, ISRAEL

Structure-based high throughput virtual screening (SBHTVS), a natural extension of molecular docking in the high-performance computing platforms, has been widely applied in lead discovery as a productive and cost-effective technology. Recently, we parallelized the well-known program DOCK developed by Kuntz et al in the supercomputers. This speeds up the database screening dramatically. Employing this approach, we have docked more than two million compounds into a series of targets, such as PPAR $\gamma, \beta$-secretase, potassium ion channel, COX-2 and tyrosine kinase. In this way, we have discovered many potent active compounds. This talk describes the SBHTVS platform in the Center for Drug Discovery and Design, Chinese Academy of Sciences, and the recent results of SBHTVS produced in this platform.

Keywords: VIRTUAL SCREENING, LEAD DISCOVERY, DRUG DESIGN
Acta Cryst. (2002). A58 (Supplement), C67

\section{A NEW FACILITY FOR THE STUDY OF MATERIALS PROCESSING ON THE SRS, DARESBURY}

$\underline{\text { R. Cernik }}^{1}$ G. Bushnell-Wye ${ }^{1}$ C . Tang ${ }^{1}$ A. Dent ${ }^{1}$ N. Terril ${ }^{1}$ G. Diakun ${ }^{1}$ P. Barnes $^{2}$ G. N. Greaves ${ }^{3}$ T. Rayment ${ }^{4}$ A. Ryan ${ }^{5}$

${ }^{1}$ Daresbury Laboratory CLRC Daresbury WARRINGTON WA4 4AD UK ${ }^{2}$ Birkbeck College London ${ }^{3}$ University of Aberystwyth ${ }^{4}$ University of Cambridge ${ }^{5}$ University of Sheffield

A new X-ray instrument, MPW6.2, is being commissioned for the study of polycrystalline and non-crystalline materials at the SRS. The beamline is optimized to receive synchrotron X-rays of 4-18 keV from a multi-pole (x 11) wiggler ( $\mathrm{B}=2$ tesla). The optics - two mirrors and a sagittal monochromator have been arranged to focus the beam vertically and horizontally respectively to deliver an estimated flux of $1011-1012$ photons $/ \mathrm{mm}^{2} / \mathrm{s} / 0.01 \%$ onto the sample under investigation, at least 100 times more intense than any other source on the SRS. At the heart of this development are two state-of-the-art detectors. The first of these is a new wide-angle curved position-sensitive detector to be mounted on a heavy-duty two-circle diffractometer. This high angular resolution $\left(0.06^{\circ}\right)$ detector system is based on the novel micro-gap multi-wire (RAPID) technology for fast data acquisition, and will allow an entire X-ray diffraction pattern (XRD) to be recorded in a very short time (milliseconds to seconds). Based on the same technology, a one-dimensional quadrant detector will also be installed for small angle X-ray scattering (SAXS). Together, these two installations constitute an exceptionally powerful instrument for combined small/wide-angle scattering (SAXS/WAXS).

Additionally, X-ray absorption apparatus will be provided for combining these techniques with spectroscopy measurements (EXAFS). Purposely designed for kinetic studies, to follow structural changes in solid and liquid state reactions and materials processing, the combination of tunable wavelength, high flux and rapid data collection make this new beamline ideal for carrying out simultaneous measurements by researchers in the field of materials science.
Acta Cryst. (2002). A58 (Supplement), C67

\section{PROBING THE LIMITS OF IN-HOUSE SAS PHASING USING FOCUSED CHROMIUM X-RAYS}

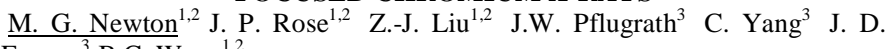
Ferrara $^{3}$ B.C. Wang ${ }^{1,2}$

University of Georgia Department of Biochemistry And Molecular Biology ${ }^{1}$ Life Science Building University of Georgia ATHENS GEORGIA 30602 USA ${ }^{2}$ Department of Biochemistry and Molecular Biology, University of Georgia, Athens, GA 30602, USA ${ }^{3}$ Rigaku/MSC, The Woodlands, TX 77381, USA

With the recent introduction of the Rigaku/MSC Chromium Confocal MaxFlux confocal optics system, researchers now have access to an intense, monochromatic, in-house Chromium $\mathrm{K} \alpha \mathrm{X}$-ray source. We have designed a series of experiments to test the feasibility of using this source to determine protein structures using single wavelength anomalous scattering (SAS) data collected on native crystals. We have shown that protein structures can be determined from single wavelength anomalous scattering data. Key to the success of this process is the signal to noise ratio $(\mathrm{s} / \mathrm{N})$ of the data used in the analysis. This is especially true for structure solution using the weak sulfur anomalous scattering signal $\left(\Delta \mathrm{f}^{\prime}=0.557\right.$ for $\mathrm{Cu}$ Ka X-rays). The use of chromium radiation $\lambda=2.29 \AA$ ) is attractive for sulfur SAS experiments since the sulfur anomalous signal is twice as strong $\left(\Delta \mathrm{f}^{\prime}=1.142\right)$ as compared to $\mathrm{Cu}$ $\mathrm{K} \alpha$. The results of these studies and the methods used to overcome problems associated with sulfur SAS data collection using the new Rigaku/MSC Chromium optics on a rotating anode X-ray source will be discussed.

Work supported in part from funds from the National Institutes of Health (GM062407), the Georgia Research Alliance, and The University of Georgia Research Foundation.

Keywords: CHROMIUM RADIATION, CONFOCAL OPTICS, SULFUR PHASING 